\title{
A NOTE ON THE CLASSES OF NON-LINEAR SEMI-SPECIAL PERMUTATIONS
}

\author{
by K. R. YACOUB
}

(Received 31st March, 1958)

In a recent paper [1], the author divided the semi-special permutations on $[n]$ that are not linear into two classes. The first class consists of the semi-special permutations which, for all possible values of $s$, have $s$ as a principal number and which induce modulo $s$ the identity permutation. The second class consists of all the semi-special permutations, with principal number $s$, which induce modulo $s$ linear permutations other than the identity, where again $s$ takes all its possible values.

Further, it was shown that no two permutations of the same class (though with different values of the parameter $s$ ) can be identical [1, Theorem 3]. It was also shown that, under certain conditions, a permutation of the first class may be identical with a permutation of the second class [1, Theorem 4]. This fact raised a question of some interest, namely, whether one of the classes is perhaps a subclass of the other. The answer to this question is, in a few cases, affirmative.

However, in some cases there exists one and only one class of such permutations. For example, if $n=2 p$, where $p$ is an odd prime, the non-linear semi-special permutations on [2 $p$ ] are of the form

$$
\pi(2 x)=2 x, \quad \pi(2 x+1) \equiv 2 x+1+2 \lambda(\bmod 2 p),
$$

where $\lambda$ is prime to $p$ [2, Theorem 4.1]. It is evident that, in this case, the permutations just described constitute only one class, namely, the first class, and the second class is in fact empty.

Furthermore, if $n=p^{2}$, where $p$ is an odd prime, the non-linear semi-special permutations on $\left[p^{2}\right]$ are of the form

$$
\pi x \equiv t x+p \mu x(x-1) \quad\left(\bmod p^{2}\right),
$$

with $t \neq 1(\bmod p)$, where $t$ and $\mu$ are both prime to $p$ and are chosen such that $u-\mu h t^{h-1}$ is also prime to $p, h$ being the order of $t$ modulo $p$, and $u$ defined modulo $p$ by $t^{h} \equiv 1+u p\left(\bmod p^{2}\right)$ [2, Theorem 4.2]. These permutations constitute again one class, namely, the second class. In this case, the first class is empty.

Nevertheless, if $n=p^{3}$ or $p^{4}$, where $p$ is an odd prime, the two classes do exist, but the first class is actually a subclass of the second [3, Theorems 8 and 9]. It is, however, interesting to see whether this fact remains true for higher powers of $p$. This is the main object of this note.

In the following paragraph, we collect the notations and results we require here.

\section{Notations and Miscellaneous Results.}

In an earlier paper [2], it was shown that if $\pi$ be a non-linear semi-special permutation on $[n]$ with principal number $s$, then it is either of the form

$$
\pi x \equiv x+s \lambda\left(1+\omega+\ldots+\omega^{x-1}\right) \quad(\bmod n),
$$

or of the form 


$$
\pi \mathrm{l}=t, \quad \pi x \equiv t x+s R \sum_{i=1}^{x-1}(x-i) \theta^{i-l} \quad(\bmod n) \quad(x \geqslant 2),
$$

where $t \not \equiv \mathbf{l}(\bmod s)$, according as the permutation induced by $\pi$ modulo $s$ is the identity permutation or is not. The parameters $\lambda, \omega, t, R$ and $\theta$ are to be chosen in the proper way [2, Theorems 3.1 and 3.10].

We remark that, when $n$ is given, the permutation $\pi$ defined by (1) depends on three parameters, namely $s, \lambda$ and $\omega$, and is denoted by $\pi(s ; \lambda, \omega)$. Furthermore, the permutation $\pi$ defined by (2) depends on four parameters, namely $s, t, R$ and $\theta$, and may therefore be denoted by $\pi(s ; t, R, \theta)$. It should be noted that the parameters $s$ and $t$ are to be determined modulo $n$, while the parameters $\lambda, \omega, R$ and $\theta$ are to be determined modulo $N$, where $N=n / s$.

Theorem 1. With the above notation, $\pi(s ; \lambda, \omega)=\pi\left(s^{\prime} ; t, R, \theta\right)$ if and only if

$$
\begin{aligned}
& s^{\prime}=k s, t \equiv 1+\lambda s \quad(\bmod n) \text {; } \\
& R \equiv \frac{\lambda(\omega-1)}{k}, \theta \equiv \omega \quad\left(\bmod N^{\prime}\right) \quad\left[k=(\omega-1, N), N=\frac{n}{s}, N^{\prime}=\frac{n}{s^{\prime}}=\frac{N}{k}\right] \\
& u+\frac{\lambda b}{s}\left\{h t^{n-1}-\frac{t^{h}-1}{t-1}\right\} \text { is prime to } N^{\prime} \\
& u k(1+\lambda s)-\lambda h(1+u k s) \equiv 0 \quad\left(\bmod N^{\prime}\right),
\end{aligned}
$$

where $h$ is the order of $t$ modulo $s^{\prime}$ and $u$ is defined modulo $N^{\prime}$ by $t^{h} \equiv 1+u s^{\prime}(\bmod n)$, and where $[1$, Theorem 4]

$$
b \equiv \frac{\omega-1}{k} \sum_{i=1}^{s-1}(s-i) \omega^{i-1} \quad\left(\bmod N^{\prime}\right)
$$

Note. It should be pointed out that conditions (5) and (6) are in fact the necessary and sufficient conditions for the existence of $\pi\left(s^{\prime} ; t, R, \theta\right)$ when $s^{\prime}, t, R$ and $\theta$ are given by (3) and (4).

Theorem 2(a). Let $p$ be an odd prime, and $\alpha>2$, and let $\lambda, t, R, \Omega$ and $\Theta$ all be prime to $p$. Then the non-linear semi-special permutations on $\left[p^{\alpha}\right]$, with principal number $p^{\beta}$, are (i)

$$
\pi x \equiv x+p^{\beta} \lambda\left(1+\omega+\ldots+\omega^{x-1}\right) \quad\left(\bmod p^{\alpha}\right),
$$

for $\beta<\alpha-1$, where $\omega \equiv 1+\Omega p^{v}\left(\bmod p^{\alpha-\beta}\right)$, with $\gamma=1, \ldots, \alpha-\beta-1$ if $2 \beta \geqslant \alpha$, and $\gamma=\alpha-2 \beta$, $\ldots, \alpha-\beta-1$ if $2 \beta<\alpha$, and (ii)

$$
\pi \mathrm{l}=t, \quad \pi x \equiv t x+p^{\beta} R \sum_{i=1}^{x-1}(x-i) \theta^{i-1} \quad\left(\bmod p^{\alpha}\right) \quad(x \geqslant 2)
$$

for $\beta \geqslant \frac{1}{2} \alpha$, where $t \not \equiv 1\left(\bmod p^{\beta}\right)$ and $\theta \equiv 1+\Theta p^{\delta}\left(\bmod p^{\alpha-\beta}\right)$ with $\delta=1, \ldots, \alpha-\beta$, and where $t, R$ and $\Theta$ are to be chosen properly [3, Theorems 5 and 6$]$.

Using the previous notation, we may write the above theorem as

Theorem 2(b). Let $n=p^{\alpha}$, where $p$ is an odd prime and $\alpha>2$, and let $\lambda, t, R, \Omega$ and $\Theta$ be chosen as in Theorem 2(a). Then the non-linear semi-special permutations on $\left[p^{\alpha}\right]$ are (i)

$$
\pi\left(p^{\beta} ; \lambda, 1+\Omega p^{\gamma}\right),
$$

for $\beta<\alpha-1$, with $\gamma=1, \ldots, \alpha \beta-1$ if $2 \beta \geqslant \alpha$, and $\gamma=\alpha-2 \beta, \ldots, \alpha-\beta-1$ if $2 \beta<\alpha$, and (ii)

$$
\pi\left(p^{\beta^{*}} ; t, R, \mathbf{1}+\Theta p^{\delta}\right) \text {, }
$$

for $\beta^{*} \geqslant \frac{1}{2} \alpha$, with $\delta=1, \ldots, \alpha-\beta$. 


\section{The Main Results.}

We start by proving the following

THEOREM 3. Let the notation be as in Theorem 2(b), and let $\beta<\alpha-1$. Then

$$
\pi\left(p^{\beta} ; \lambda, 1+\Omega p^{\gamma}\right)=\pi\left(p^{\beta^{*}} ; 1+\lambda p^{\beta}, \lambda \Omega, 1+\Omega p^{\gamma}\right),
$$

where $\beta^{*}=\beta+\gamma$.

Proof. Suppose that

$$
\pi\left(p^{\beta} ; \lambda, 1+\Omega p^{\nu}\right)=\pi(s ; t, R, \theta) ;
$$

then, by Theorem 1, we have

because $\Omega$ is prime to $p$,

$$
s=\left(\Omega p^{\gamma}, p^{\alpha-\beta}\right) \times p^{\beta}=p^{\beta+\gamma}=p^{\beta^{*}},
$$

$$
t \equiv 1+\lambda p^{\beta} \quad\left(\bmod p^{\alpha}\right),
$$

and

$$
R \equiv \lambda \Omega, \quad \theta \equiv 1+\Omega p^{\gamma} \quad\left(\bmod p^{\alpha-\beta^{*}}\right) .
$$

It remains to show that with these values of $s, t, R$ and $\theta$, conditions (5) and (6) are satisfied identically. Here $h$ is the order of $t$ modulo $s$, where $t \equiv 1+\lambda p^{\beta}\left(\bmod p^{\alpha}\right)$ and $s=p^{\beta^{*}} ;$ also $u$ is defined modulo $p^{\alpha-\beta^{*}}$ by $t^{h}=1+u p^{\beta^{*}}\left(\bmod p^{\alpha}\right)$.

Now, since $t \equiv 1+\lambda p^{\beta}\left(\bmod p^{\alpha}\right)$ and $\lambda$ is prime to $p$, it follows that $h=p^{\gamma}$, and then $u \equiv \lambda\left(1+U p^{\beta}\right)\left(\bmod p^{\alpha-\beta^{*}}\right)$ for some integer $U$. Also

$$
\begin{aligned}
h t^{h-1}-\frac{t^{h}-1}{t-1} & \equiv p^{\gamma}\left(1+\lambda p^{\beta}\right)^{p^{\gamma}-1}-\sum_{i=1}^{p^{\gamma}}\left(\begin{array}{c}
p^{\gamma} \\
i
\end{array}\right)\left(\lambda p^{\beta}\right)^{i-1} \quad\left(\bmod p^{\alpha}\right) \\
& \equiv p^{\beta+\gamma T} \quad\left(\bmod p^{\alpha}\right)
\end{aligned}
$$

for some integer $T$. Condition (5) then requires that

$$
\lambda\left(1+U p^{\beta}\right)+\lambda b p^{\nu} T
$$

is prime to $p$, which is already secured since $\lambda$ is prime to $p$ [see Theorems $2(b)$ and $2(a)$ ]. Moreover, condition (6) reduces to

$$
\lambda\left(1+U p^{\beta}\right) p^{\gamma}\left(1+\lambda p^{\beta}\right)-\lambda p^{\nu}\left\{1+\lambda\left(1+U p^{\beta}\right) p^{\gamma+\beta}\right\} \equiv 0 \quad\left(\bmod p^{\alpha-\beta^{*}}\right)
$$

i.e. to

$$
\lambda p^{\beta^{*}}\left\{U+\lambda+U \lambda p^{\beta}-\lambda\left(1+U p^{\beta}\right) p^{\nu}\right\} \equiv 0 \quad\left(\bmod p^{\alpha-\beta^{*}}\right) .
$$

Condition (7) is secured if $2 \beta^{*} \geqslant \alpha$.

We now show that $2 \beta^{*} \geqslant \alpha$. For if $\beta \geqslant \frac{1}{2} \alpha$, we have $2 \beta^{*}=2 \beta+2 \gamma>2 \beta>\alpha$; also when $\beta<\frac{1}{2} \alpha, \gamma$ takes one of the values $\alpha-2 \beta, \ldots, \alpha-\beta-1$ and thus $2 \beta^{*}>\alpha$. Hence condition (6) is, for all $\beta<\alpha-1$, secured and the theorem is proved.

Theorem 3 leads at once to the following theorem.

THEOREM 4. Let $p$ be an odd prime and $\alpha>2$. Then the two classes of non-linear semispecial permutations on $\left[p^{\alpha}\right]$ are both non-empty. Moreover the first class is always a subclass of the second.

When $\alpha=2$, the first class is empty and the second part of the theorem is trivial. 


\section{REFERENCES}

1. K. R. Yacoub, A note on semi-special permutations, Proc. Glasgow Math. Assoc., 3 (1958), 164-169.

2. K. R. Yacoub, On semi-special permutations I, Proc. Glasgow Math. Assoc., 3 (1956), 18-35.

3. K. R. Yacoub, On semi-special permutations IT. Semi-special permutations on $\left[p^{\alpha}\right]$. Duke Math.J., 24 (1957), 455-465.

Faculty of Sonence

ALEXANDRIA UNIVERSTTY

EGYPT 